\title{
PERSPECTIVE OPEN Cdk4/6 inhibitors and overall survival: power of first-line trials in metastatic breast cancer
}

\author{
Marie-Laure Tanguy ${ }^{1}$, Luc Cabel ${ }^{2,3}$, Fréderique Berger ${ }^{1}$, Jean-Yves Pierga ${ }^{2,4}$, Alexia Savignoni ${ }^{1}$ and Francois-Clement Bidard iD $^{2,3}$
}

Palbociclib, ribociclib, and abemaciclib have been investigated in combination with aromatase inhibitors as first-line therapy for metastatic hormone receptor-positive breast cancer (PALOMA-2, MONALEESA-2 and MONALEESA-7, MONARCH-3 trials, respectively); pivotal trials led to absolute median progression-free survival (PFS) gain of about 15 months. We aimed to estimate, for each trial, the statistical power to demonstrate a significant gain in overall survival (OS). Power was calculated with Freedman's formula. Given the allocation ratio and the number of events, power was computed as a function of hazard ratio. We focused on four specific hazard ratio values $(0.94,0.89,0.81$, and 0.77$)$, which are estimated to correspond to absolute $3,6,12$, and 15 months gain in OS, respectively. For these calculations, the type I error rate was stated at $5 \%$ with a two-sided test, and we assumed that the risk of death was constant over time. PALOMA-2 and MONALEESA trials have an almost similar power despite different allocation ratios, while MONARCH-3 has a more limited power. Overall, the power of the four trials to demonstrate a statistically significant improvement in OS is less than $70 \%$ if the prolongation in median OS is $\leq 12$ months, whatever the OS data maturity. This analysis shows that OS results are jeopardized by limited powers, and a meta-analysis might be required to demonstrate OS benefit. Conversely, if a significant OS improvement is observed in some but not at all trials, this discrepancy might be more attributable to chance than to a truly different drug efficacy.

npj Breast Cancer (2018)4:14; doi:10.1038/s41523-018-0068-4

\section{INTRODUCTION}

Endocrine therapies are the cornerstone of hormone receptorpositive $(\mathrm{HR}+$ ) HER2-negative (HER2-) breast cancer treatment at both early and metastatic stages. Endocrine therapies for metastatic breast cancer (MBC) have remained largely unchanged for the past two decades, and include tamoxifen, aromatase inhibitors (Al), and fulvestrant. ${ }^{1}$ In 2012, results of BOLERO-2, a randomized placebo-controlled phase 3 conducted in patients with $\mathrm{HR}+\mathrm{HER} 2-\mathrm{MBC}$ progressing under first-line nonsteroidal Al, have been reported. ${ }^{2}$ This trial compared the efficacy of a steroidal Al (exemestane) to that of a combination of exemestane and everolimus, a mTOR inhibitor. Patients in the everolimusexemestane arm had a significantly longer PFS, with a hazard ratio $(\mathrm{HR})=0.43,95 \% \mathrm{Cl}[0.35 ; 0.54]^{2}$ In that second-line setting, despite a 4.6-month prolongation in median PFS, adding everolimus to exemestane did not confer a statistically significant improvement in the overall survival (OS): $\mathrm{HR}=0.89,95 \% \mathrm{Cl}[0.73$; $1.10] .^{3}$ This negative result increased the concerns about the limited cost-effectiveness of everolimus in that setting. ${ }^{4,5}$

More recently, further significant progresses have been reported in HR+ HER2-MBC: four randomized phase 3 trials have reported superior progression-free survivals (PFS) for Al and cdk4/6 inhibitors combinations compared to Al and placebo as first-line therapy. The PALOMA-2 trial, in which 666 patients have been randomized 2:1 between the Al and palbociclib arm and the $\mathrm{Al}$ and placebo arm, was the first to be reported and demonstrated a PFS HR of $0.58,95 \% \mathrm{Cl}[0.46 ; 0.72] .{ }^{6}$ In the MONALEESA-2 trial, 668 patients have been randomized in a 1:1 fashion between the $\mathrm{Al}$ and ribociclib arm and the Al and placebo arm, with a PFS $\mathrm{HR}$ of $0.56,95 \% \mathrm{Cl}[0.43 ; 0.72] .^{7}$ Superimposable number of included patients and results have been reported with ribociclib in a second pivotal trial, MONALEESA-7, that was conducted in premenopausal women [8]. Recently, in the MONARCH-3 trial, 493 patients have been randomized in a 2:1 fashion between the $\mathrm{Al}$ and abemaciclib arm and the Al and placebo arm, with a PFS HR of $0.54,95 \% \mathrm{Cl}[0.41 ; 0.72]^{8}$ (Table 1). Based on these significant PFS improvements, cdk4/6 inhibitors have been approved by regulatory agencies for first-line HR+ HER2- MBC and are now being largely used in that setting. However, in the context of a metastatic disease, and not withstanding quality of life-related endpoints, the ultimate goal of a palliative therapy is to extend OS, while PFS is moderately correlated with OS. $^{9}$ In all three trials, OS was defined as a secondary endpoint, and no mature data have been reported so far ( $20 \%$ of deaths were observed in the last MONALEESA-2). ${ }^{10}$

Per protocol, 278 OS events (41\% maturity) and 315 OS events (47\% maturity) will trigger the main OS analysis in PALOMA-2 and MONALEESA-2, respectively. In the MONARCH-3 trial, the main OS analysis is planned as part of a pooled analysis with the MONARCH-2 study; OS analyses of MONARCH-3 as a single study could be reported as exploratory analyses with no prespecified maturity. As for many trials and despite methodological concerns, it is very likely that unplanned OS analyses will be reported even after the main analyses have occurred.

In this report, we estimated the power of each of the three trials to demonstrate a significant gain in OS according to their intrinsic

\footnotetext{
${ }^{1}$ Department of Biometry, Institut Curie, PSL Research University, Saint Cloud, France; ${ }^{2}$ Department of Medical Oncology, Institut Curie, PSL Research University, Paris, France; ${ }^{3}$ UVSQ, Paris Saclay University, Saint Quentin en Yvelines, Paris, France and ${ }^{4}$ Paris Descartes University, Paris, France

Correspondence: F-C. Bidard (fcbidard@curie.fr)
}

Received: 28 January 2018 Revised: 3 June 2018 Accepted: 4 June 2018

Published online: 26 June 2018 
Table 1. Summary of trial characteristics and results

\begin{tabular}{|c|c|c|c|c|}
\hline & PALOMA-2 trial & MONALEESA-2 trial & MONALEESA-7 trial & MONARCH-3 trial \\
\hline cdk4/6inh & Palbociclib & Ribociclib & Ribociclib & Abemacicl \\
\hline Number of patients $\mathrm{Al}+$ placebo arm & 222 & 334 & 337 & 165 \\
\hline Median PFS Al + placebo arm & 14.5 months & 14.7 months & 13 months & 14.7 months \\
\hline Number of patients $\mathrm{Al}+\mathrm{cdk} 4 / 6 \mathrm{inh}$ arm & 444 & 334 & 337 & 328 \\
\hline Median PFS Al + cdk4/6inh arm & 24.8 months & Not reached & 23.8 months & Not reached \\
\hline PFS hazard ratio $[95 \% \mathrm{Cl}]$ & $0.58[0.46 ; 0.72]$ & $0.56[0.43 ; 0.72]$ & $0.55^{\mathrm{a}}$ & $0.54[0.41 ; 0.72]$ \\
\hline
\end{tabular}

design (number of patients included, randomization ratio), and the number of events.

\section{RESULTS}

Figure 1 depict, for each OS data maturity and for each of the three phase 3 trial, the power to demonstrate a statistically significant improvement in OS according to different HRs. As expected, the higher the OS maturity, the higher the power is for a given HR. Unsurprisingly, MONALEESA-2 and -7, which share similar design and number of treated patients, have superimposable powers. We observed that PALOMA-2 and MONALEESA trials have an almost similar power, MONALEESA trials displaying a marginally increased power. We confirm that the MONARCH-3 trial is less powerful than the three other trials.

For each of the four specific HR values proposed, the power of each trial is displayed on Table 2. Whatever the OS data maturity, if the prolongation in median OS is less than 12 months, the four trials have a power less than $70 \%$. For a prolongation of 15 months, a power of $80 \%$ or more is reached for a death rate of $80 \%$. The power of MONARCH-3 is about $72 \%$.

\section{DISCUSSION}

In the absence of cure, the ultimate goals of metastatic cancer therapy are to improve duration and/ or quality of survival of the patients. New anticancer drugs should therefore demonstrate a benefit on OS and/or an improvement of the quality of life. Other clinical endpoints such as PFS may be useful intermediates, but are not surrogates of $O S$ and, in $M B C$, the level of evidence supporting a surrogacy of any biomarker, ${ }^{9}$ including circulating tumor cells count is low. ${ }^{11}$

Here we showed that MONARCH-3 is less powerful than PALOMA-2, MONALEESA-2, and MONALEESA-7 trials, and that these three latter trials have an almost similar power; the small difference in power between them is explained by the difference in treatment allocation ratio: 2:1 in the PALOMA-2 trial vs 1:1 in the MONALEESA trials, the three trials having enrolled a similar number of patients.

PALOMA-2 and MONALEESA trials have a $<0.70$ power to report a statistical difference if the observed absolute PFS gain (about 15 months, in first line) is translated into a 12-month OS improvement; smaller OS improvements will be more difficult to demonstrate. A power of $80 \%$ is observed only if the OS improvement is 15 months or more. Depending on the final OS HR achieved by cdk4/6 inhibitors, the more limited power of MONARCH-3 may be responsible for a scenario in which a statistically significant OS gain is observed in PALOMA-2 and MONALEESA trials, but not in MONARCH-3-even if there is no true efficacy difference among the three cdk4/6 inhibitors. Our analysis illustrate that, OS being the preferred outcome, it will not be satisfactory when median OS is long and differences need to be very large to demonstrate an effect. Such comparisons are further complicated by the numerous post-progression therapies available in $\mathrm{MBC}$, which will make the two arms more similar. A gain in OS is much more likely to be demonstrated by pooling individual patient data from different trials. A pooled analysis of MONALEESA-2 and MONALEESA-7 trials will be probably be reported in a near future, as both trials tested the same drug. A larger meta-analysis including the individual data from all patients including the four trials would have a very high statistical power to demonstrate that cdk4/6 inhibitor do increase OS. If not made mandatory by regulatory agencies, the feasability of such metaanalysis will rely on the willingness of three competing pharmaceutical companies.to collaborate.

Our analyses have several limitations, as many parameters (post-first line therapies, treatment with cdk4/6 inhibitors at a later stage...) will ultimately influence the OS differences between the two arms of each trial and between trials. A key parameter will be the OS reached by control arms, which is currently unknown. The PALOMA-2 study hypothesized that a median OS of 34 months in the control arm of this international trial, while our analyses were based on real life OS data obtained in France in that specific population of Al-sensitive metastatic MBC patients (50 months).

In conclusion, power to demonstrate a gain in OS limited in these trials; ultimately, if a significant OS improvement is observed in some but not at all trials, this discrepancy might be more attributable to chance than to a truly different drug impact on OS.

\section{METHODS}

Power was calculated with Freedman's formula, which allows us to compute power for studies comparing survivor functions of two groups by using the log-rank test. Given the allocation ratio and the number of events, power was computed as a function of HR. For these calculations, the type I error rate was stated at $5 \%$ with a two-sided test, and we assumed that the risk of death was constant over time. We particularly focused on four hypothetic prolongations in median OS following the use of cdk4/6 inhibitors: $3,6,12$, and 15 months. To translate HR into clinically meaningful data, we hypothesized that the median OS in control arms will be equal to 50 months, as observed in more than $6000 \mathrm{Al}$-sensitive HR + HER2 - MBC patients treated prior to the cdk4/6 inhibitor era. ${ }^{12}$ According to the prolongations proposed above, the four HR of interest are $0.94,0.89,0.81$, and 0.77 , respectively. We calculated the power for two hypotheses of death rates occurring over the follow-up period: 40,60 , and $80 \%$, reflecting the OS data maturity. The analysis was performed using $\mathrm{R}$ software version 3.3.2 [13].

Data availability statement

All data generated or analyzed during this study are included in this published article. 
a
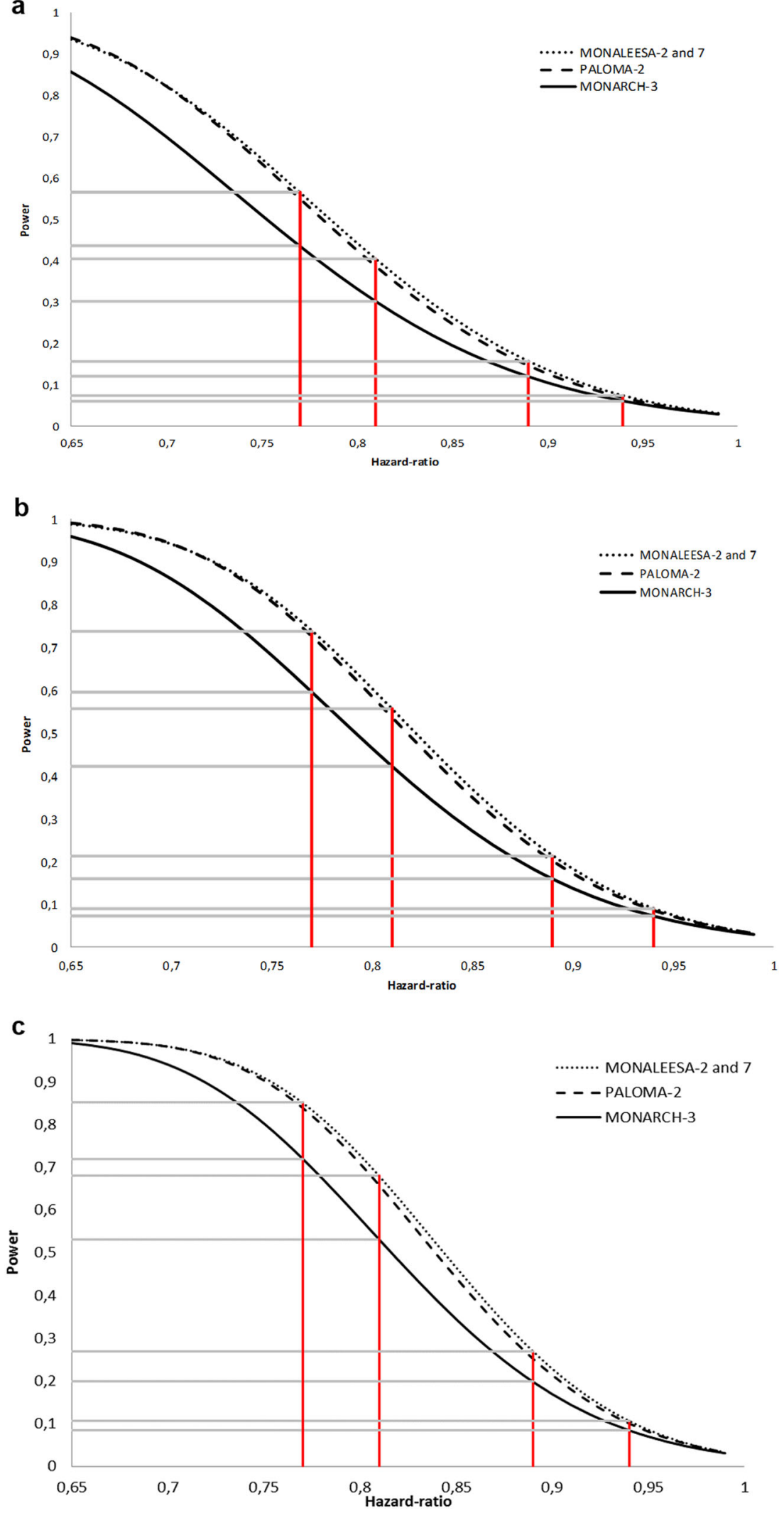

Fig. 1 Power to demonstrate a benefit on overall survival. a-c Power at 40,60 , and $80 \%$ death rate (maturity), respectively. HR of 0.94 , 0.89 , $0.81,0.77$, which are estimated to correspond to absolute $3,6,12$, and 15 months gain in OS (on the basis of a 50 months median OS in control $\mathrm{arms}^{12}$ ) are highlighted in red 
Table 2. Examples of power estimates

\begin{tabular}{llllll}
\hline Hazard ratios & Death rate & PALOMA-2 trial & MONALEESA-2 trial & MONALEESA-7 trial & MONARCH-3 trial \\
\hline $\mathbf{0 . 7 7}$ & $40 \%$ & 0.55 & 0.57 & 0.57 & 0.44 \\
(15 months gain in median OS) & $60 \%$ & 0.73 & 0.74 & 0.74 & 0.85 \\
& $80 \%$ & 0.84 & 0.85 & 0.40 & 0.72 \\
$\mathbf{0 . 8 1}$ & $40 \%$ & 0.39 & 0.40 & 0.56 & 0.30 \\
(12 months gain in median OS) & $60 \%$ & 0.54 & 0.56 & 0.68 & 0.42 \\
& $80 \%$ & 0.66 & 0.68 & 0.16 & 0.53 \\
$\mathbf{0 . 8 9}$ & $40 \%$ & 0.15 & 0.16 & 0.21 & 0.12 \\
(6 months gain in median OS) & $60 \%$ & 0.20 & 0.21 & 0.27 & 0.16 \\
& $80 \%$ & 0.25 & 0.27 & 0.07 & 0.20 \\
$\mathbf{0 . 9 4}$ & $40 \%$ & 0.07 & 0.07 & 0.09 & 0.06 \\
(3 months gain in median OS) & $60 \%$ & 0.09 & 0.09 & 0.11 \\
\end{tabular}

Calculations were made on the basis of a 50 months median OS in control arms [12]. The power values displayed in this table are also displayed Fig. 1

\section{ACKNOWLEDGEMENTS}

This study was funded by Institut Curie SIRIC 2 (grant INCa-DGOS-Inserm_12554).

\section{AUTHOR CONTRIBUTIONS}

All authors researched, collated, and wrote this paper.

\section{ADDITIONAL INFORMATION}

Competing interests: F.-C. Bidard is part of advisory boards and received research grants (unrelated to this study) from Pfizer, Novartis, and Lilly. Other authors have no competing interest.

Publisher's note: Springer Nature remains neutral with regard to jurisdictional claims in published maps and institutional affiliations.

\section{REFERENCES}

1. Cardoso, F. et al. 3rd ESO-ESMO International Consensus Guidelines for Advanced Breast Cancer (ABC 3). Ann. Oncol. mdw544 (2016). https://doi.org/ 10.1093/annonc/mdw544

2. Baselga, J. et al. Everolimus in postmenopausal hormone-receptor-positive advanced breast cancer. N. Engl. J. Med. 366, 520-529 (2012).

3. Piccart, M. et al. Everolimus plus exemestane for hormone-receptor-positive, human epidermal growth factor receptor-2-negative advanced breast cancer: overall survival results from BOLERO-2†. Ann. Oncol. Off. J. Eur. Soc. Med. Oncol. 25, 2357-2362 (2014).

4. Niraula, S., Ocana, A. \& Amir, E. One step forward, two steps back: the story of everolimus in advanced breast cancer. Breast Edinb. Scotl. 24, 529-531 (2015).

5. Diaby, V. et al. Cost-effectiveness analysis of everolimus plus exemestane versus exemestane alone for treatment of hormone receptor positive metastatic breast cancer. Breast Cancer Res. Treat. 147, 433-441 (2014).
6. Finn, R. S. et al. Palbociclib and letrozole in advanced breast cancer. N. Engl. J. Med. 375, 1925-1936 (2016)

7. Hortobagyi, G. N. et al. Ribociclib as first-line therapy for HR-positive, advanced breast cancer. N. Engl. J. Med. 375, 1738-1748 (2016).

8. Goetz, M. P. et al. MONARCH 3: abemaciclib as initial therapy for advanced breast cancer. J. Clin. Oncol. Off. J. Am. Soc. Clin. Oncol. 35, 3638-3646 (2017).

9. Fiteni, F. \& Bonnetain, F. Surrogate end points for overall survival in breast cancer trials: a review. Breast 29, 44-48 (2016).

10. Hortobagyi, G. N. et al. Updated results from MONALEESA-2, a phase 3 trial of first-line ribociclib+letrozole in hormone receptor-positive (HR+), HER2negative (HER2-), advanced breast cancer (ABC). J. Clin. Oncol. 35, 1038-1038 (2017).

11. Cabel, L. et al. Circulating tumor cells: clinical validity and utility. Int. J. Clin. Oncol. 22, 421-430 (2017).

12. Le Saux, O. et al. Assessment of multiple endocrine therapies for metastatic breast cancer in a multicenter national observational study. J. Clin. Oncol. 35, 1052-1052 (2017).

cc) Open Access This article is licensed under a Creative Commons Attribution 4.0 International License, which permits use, sharing, adaptation, distribution and reproduction in any medium or format, as long as you give appropriate credit to the original author(s) and the source, provide a link to the Creative Commons license, and indicate if changes were made. The images or other third party material in this article are included in the article's Creative Commons license, unless indicated otherwise in a credit line to the material. If material is not included in the article's Creative Commons license and your intended use is not permitted by statutory regulation or exceeds the permitted use, you will need to obtain permission directly from the copyright holder. To view a copy of this license, visit http://creativecommons. org/licenses/by/4.0/.

(c) The Author(s) 2018 